Document downloaded from:

http://hdl.handle.net/10251/120657

This paper must be cited as:

Bernabeu Jiménez, T.; Valero-Nogueira, A.; Vico Bondía, F.; Kishk, AA. (2018). On the Contribution to the Field of the Nonphysical Characteristic Modes in Infinite Dielectric Circular Cylinders Under Normal Excitation. IEEE Transactions on Antennas and Propagation. 66(1):505-510. https://doi.org/10.1109/TAP.2017.2768581

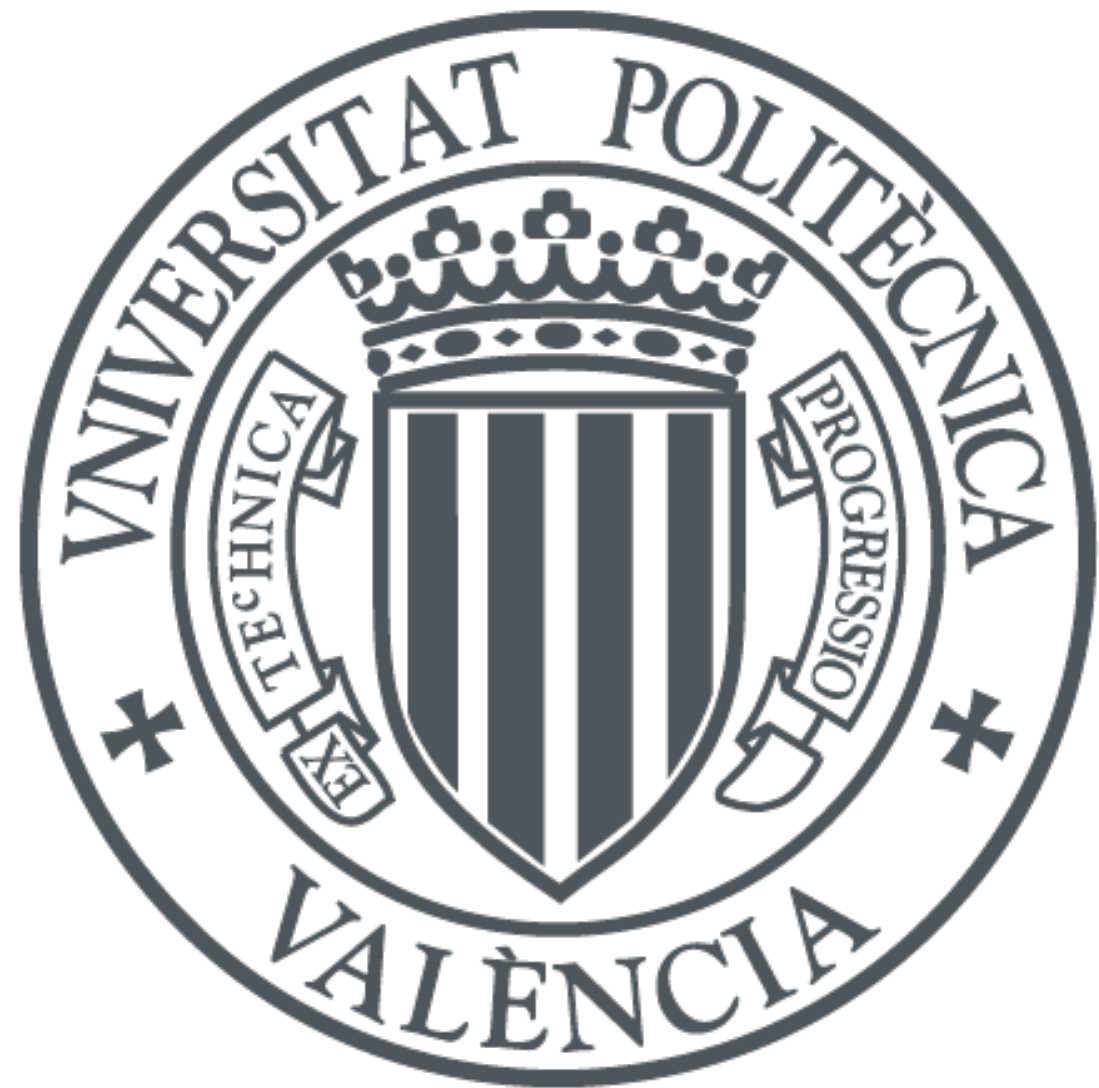

The final publication is available at

http://doi.org/10.1109/TAP.2017.2768581

Copyright Institute of Electrical and Electronics Engineers

Additional Information

(c) 2018 IEEE. Personal use of this material is permitted. Permission from IEEE must be obtained for all other users, including reprinting/ republishing this 


\section{On the Contribution to the Field of the Non-Physical Characteristic Modes in Infinite Dielectric Circular Cylinders under Normal Incidence}

\author{
Tomás Bernabeu-Jiménez, Member, IEEE, \\ Alejandro Valero-Nogueira, Senior Member, IEEE, \\ Felipe Vico-Bondia, Member, IEEE, \\ and Ahmed A. Kishk, Fellow, IEEE
}

\begin{abstract}
Here, a detailed analysis of characteristic modes and fields of an infinite dielectric circular cylinder when computed through the PMCHWT formulation is carried out. The purpose is to determine their contribution to the total field, inside and outside the dielectric body and under two possible excitations: incident plane wave or electric line source within the cylinder. The study has been done analytically to provide necessary physical insight of the results obtained. New details about socalled non-physical modes are provided. It is found that these modes, that can be neglected outside the dielectric body, do have a significant contribution to the inner field when the excitation source is within the dielectric body. It is concluded that the terms physical and non-physical characteristic modes should more properly be replaced for radiating and non-radiating characteristic modes.
\end{abstract}

Keywords-Characteristic modes, dielectric cylinders, non-physical modes, PMCHWT formulation.

\section{INTRODUCTION}

Since the Theory of Characteristic Modes (TCM) was proposed in 1968, it has been increasingly used for antenna design and extended to other fields in electromagnetics. This theory was first formulated by Garbacz for conducting bodies of arbitrary shape through the scattering matrix diagonalization [1]. Conceptually speaking, Garbacz was the first to show that there exists a modal representation acting as a real Hilbert space basis. Later, Harrington, Mautz and Chang reformulated this theory establishing a more direct method to obtain Garbacz's modal expansion [2]-[4]. They considered integrodifferential formulations using the impedance matrix of the Method of Moments (MoM).

For the past ten years, mainstream interest in TCM has been focused on conducting bodies [5]-[7]. Recently, however, researchers are turning to more complex structures including dielectrics, such as dielectric resonator antennas (DRAs), for instance [8]-[14]. For both, conducting and dielectric bodies it is important to find those electromagnetic modes that provide the desired radiation characteristics. However, with regard to dielectric bodies, there are still unknown aspects in the interpretation of resulting eigensolutions. Difficulties arise in the understanding of the solutions using surface integral equations (SIE) based on Poggio-Miller-Chang-Harrington-Wu-Tsai (PMCHWT) formulation [15]. Precisely, this SIE formulation provides two eigenvalues for each mode. In [10] and [16], it appeared that some of the solutions did not fulfill the orthonormality relation of the characteristic fields proposed by Chang and Harrington in [17]. Furthermore, given the increasing number of applications of the TCM they advised to avoid these solutions. These solutions were regarded as non-physical solutions. Even if these non-physical solutions do not satisfy the orthonormality condition for the characteristic fields, it is not demonstrated that their contribution can be discarded for the total field within the dielectric body. Furthermore, the role of the source location in the excitation of these modes has not been fully covered. Thus, depending on the excitation considered, wire source within the dielectric body or incident plane-wave, the non-physical solutions may or may not be excited.

Here, the research carried out concerns mainly the physical interpretation of the characteristic modes of an infinite dielectric circular

Manuscript received July 27, 2015; revised March 2, 2016. cylinder when a PMCHWT integro-differential formulation is used. Given the canonical nature of the problem, the analysis leads to explicit analytical solutions, providing faster solutions and deeper knowledge than a purely numerical analysis. In addition, this analytical solution will serve as a reference to compare with numerical MoM solutions based on SIE. As part of the analysis, the contribution of each eigensolution to the total electric and magnetic equivalent currents is discussed. This discussion is carried out considering two situations: a normally incident $T M^{z}$ plane wave and an electric line source located inside the cylinder. This will help to clarify the role of all characteristic modes provided by the PMCHWT formulation.

Given the purpose of this communication, the theoretical development leading to the analytical matrix operators has not been included for brevity. Moreover, only the infinite dielectric circular cylinder is considered for simplicity, considering only those modes that can be excited under normal incidence. Other geometries are expected to be studied in future work.

Notice that since the objective of this work is to demonstrate that the so-called non-physical modes can contribute to the electromagnetic field, it will be enough to find a case for which these modes contribute to the total field. Thus forcing us to rethink the physical interpretation of these modes. Therefore, the simplest case of a centered line source in the middle of the dielectric circular cylinder will suffice to this purpose.

\section{CHARACTERISTIC MODES OF INFINITE DIELECTRIC CIRCULAR CYLINDERS}

Let us directly consider the integro-differential matrix operators in (1) for $T M^{z}$, and in (2) for $T E^{z}$, where $a$ is the cylinder radius, $H_{n}^{(2)}(x)$ are the Hankel functions of the second kind, $J_{m}(x)$ the Bessel functions of the first kind, and $k_{1}$ and $k_{2}$ the outer and inner wavenumbers, respectively.

These operators have been obtained analytically for an infinite dielectric cylinder of circular cross-section using the PMCHWT formulation used by Chang and Harrington in [4]. Furthermore, since the infinite circular cylinder is a canonical geometry, it is noteworthy to mention that $n$ index dependence in matrix operators (1) and (2) implies orthogonality between the $n$ modes and they can be solved independently from each other. The resulting matrix equation in [4] is repeated in (3) for clarity,

$$
\left[\begin{array}{cc}
L_{e} & N \\
N & L_{m}
\end{array}\right]\left[\begin{array}{c}
\mathbf{J} \\
j \mathbf{M}
\end{array}\right]_{\tan }=\left[\begin{array}{c}
\mathbf{E}^{i n c} \\
j \mathbf{H}^{i n c}
\end{array}\right]_{\tan }
$$

where $\mathbf{E}^{i n c}$ and $\mathbf{H}^{\text {inc }}$ are the incident fields, $\mathbf{J}$ and $\mathbf{M}$ are the total electric and magnetic surface equivalent currents, $[\cdot]_{\tan }$ means tangential component of the bracketed quantity on the cylinder surface, and $L_{e}, L_{m}$ and $N$ are the operators involved in the PMCHWT formulation. The imaginary number $j$ is an added factor employed to make the matrix operator symmetric. For the sake of brevity, vectors in (3) can be grouped as

$$
\mathbf{f}=\left[\begin{array}{c}
\mathbf{J} \\
j \mathbf{M}
\end{array}\right] \quad \mathbf{g}=\left[\begin{array}{c}
\mathbf{E}^{i n c} \\
j \mathbf{H}^{i n c}
\end{array}\right]
$$

and the matrix operator as

$$
T=\left[\begin{array}{cc}
L_{e} & N \\
N & L_{m}
\end{array}\right]
$$

Therefore equation (3) can be written as

$$
T(\mathbf{f})=\mathbf{g}^{i}
$$




$$
\begin{gathered}
T_{n}^{T M^{z}}=(\pi a)^{2}\left[\begin{array}{cc}
\omega\left(\mu_{2} J_{n}\left(k_{2} a\right) H_{n}^{(2)}\left(k_{2} a\right)+\mu_{1} J_{n}\left(k_{1} a\right) H_{n}^{(2)}\left(k_{1} a\right)\right) & \left(k_{2} J_{n}^{\prime}\left(k_{2} a\right) H_{n}^{(2)}\left(k_{2} a\right)+k_{1} J_{n}\left(k_{1} a\right) H_{n}^{\prime(2)}\left(k_{1} a\right)\right) \\
\left(k_{2} J_{n}^{\prime}\left(k_{2} a\right) H_{n}^{(2)}\left(k_{2} a\right)+k_{1} J_{n}\left(k_{1} a\right) H_{n}^{\prime(2)}\left(k_{1} a\right)\right) & \omega\left(\epsilon_{2} J_{n}^{\prime}\left(k_{2} a\right) H_{n}^{\prime(2)}\left(k_{2} a\right)+\epsilon_{1} J_{n}^{\prime}\left(k_{1} a\right) H_{n}^{\prime(2)}\left(k_{1} a\right)\right)
\end{array}\right] \\
T_{n}^{T E^{z}}=(\pi a)^{2}\left[\begin{array}{cc}
\omega\left(\mu_{2} J_{n}^{\prime}\left(k_{2} a\right) H_{n}^{\prime(2)}\left(k_{2} a\right)+\mu_{1} J_{n}^{\prime}\left(k_{1} a\right) H_{n}^{\prime(2)}\left(k_{1} a\right)\right) & \left(k_{2} J_{n}^{\prime}\left(k_{2} a\right) H_{n}^{(2)}\left(k_{2} a\right)+k_{1} J_{n}\left(k_{1} a\right) H_{n}^{\prime(2)}\left(k_{1} a\right)\right) \\
\left(k_{2} J_{n}^{\prime}\left(k_{2} a\right) H_{n}^{(2)}\left(k_{2} a\right)+k_{1} J_{n}\left(k_{1} a\right) H_{n}^{\prime(2)}\left(k_{1} a\right)\right) & \omega\left(\epsilon_{2} J_{n}\left(k_{2} a\right) H_{n}^{(2)}\left(k_{2} a\right)+\epsilon_{1} J_{n}\left(k_{1} a\right) H_{n}^{(2)}\left(k_{1} a\right)\right)
\end{array}\right]
\end{gathered}
$$

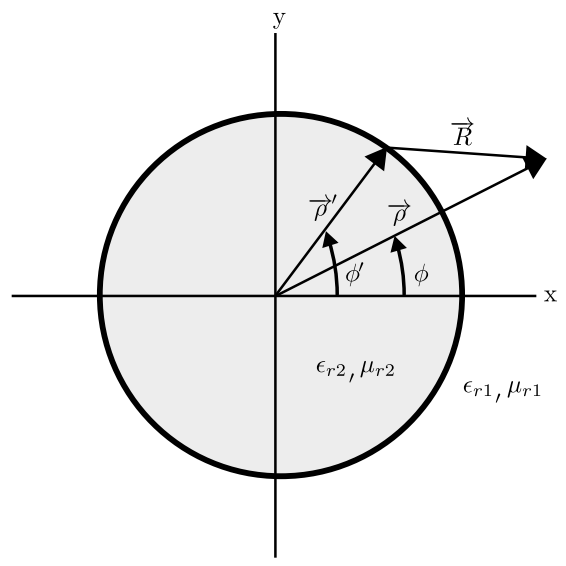

Fig. 1: Geometry and material of the considered infinite dielectric circular cylinder

where subscript tan has been dropped for brevity. See [4] for more details about the derivation of operator (5).

The dielectric circular cylinder is shown in Fig. 1. The pairs $(\rho, \phi)$ and $\left(\rho^{\prime}, \phi^{\prime}\right)$ are observation and source point locations in polar coordinates, respectively. Pairs $\left(\epsilon_{r_{1}}, \mu_{r_{1}}\right)$ and $\left(\epsilon_{r_{2}}, \mu_{r_{2}}\right)$ are the relative permittivity and permeability for outer and inner media, respectively.

The characteristic eigenvalues of a given $n$-th mode are calculated solving the weighted eigenvalue problem (7) on the $T_{n}$ operators (1) and (2). Since $T_{n}$ is symmetric, it can be expressed in terms of its Hermitian parts as $T_{n}=T_{n, r e}+j T_{n, i m}$, and the eigenvalue equation defining the characteristic modes is

$$
\left[T_{n, i m}\right]\left[\mathbf{f}_{n}\right]=\lambda_{n}\left[T_{n, r e}\right]\left[\mathbf{f}_{n}\right]
$$

In (7), $\lambda_{n}$ are real characteristic eigenvalues and $\mathbf{f}_{n}$ the coefficients of the characteristic cylindrical modes $\{\cos (n \phi), \sin (n \phi)\}$, or characteristic modes. The characteristic modes are normalized to radiate unit power. The usual orthogonality relationships are expressed as

$$
\begin{aligned}
& \left\langle\mathbf{f}_{m}, T_{n}\left(\mathbf{f}_{n}\right)\right\rangle=\left\langle\mathbf{f}_{m}^{*}, T_{n}\left(\mathbf{f}_{n}\right)\right\rangle=\left(1+j \lambda_{n}\right) \delta_{m, n} \\
& \left\langle\mathbf{f}_{m}, T_{n, r e}\left(\mathbf{f}_{n}\right)\right\rangle=\left\langle\mathbf{f}_{m}^{*}, T_{n, r e}\left(\mathbf{f}_{n}\right)\right\rangle=\delta_{m, n} \\
& \left\langle\mathbf{f}_{m}, T_{n, i m}\left(\mathbf{f}_{n}\right)\right\rangle=\left\langle\mathbf{f}_{m}^{*}, T_{n, i m}\left(\mathbf{f}_{n}\right)\right\rangle=\lambda_{n} \delta_{m, n}
\end{aligned}
$$

These equations show that the eigenvectors form a weighted orthogonal set over the material body surface.

As it will be discussed later, since the total electric and magnetic current distributions are function of their characteristic currents, it is useful to write equation (7) as a function of their electric and magnetic characteristic currents, $J_{n}^{\lambda_{n}^{(i)}}$ and $M_{n}^{\lambda_{n}^{(i)}}$ respectively.

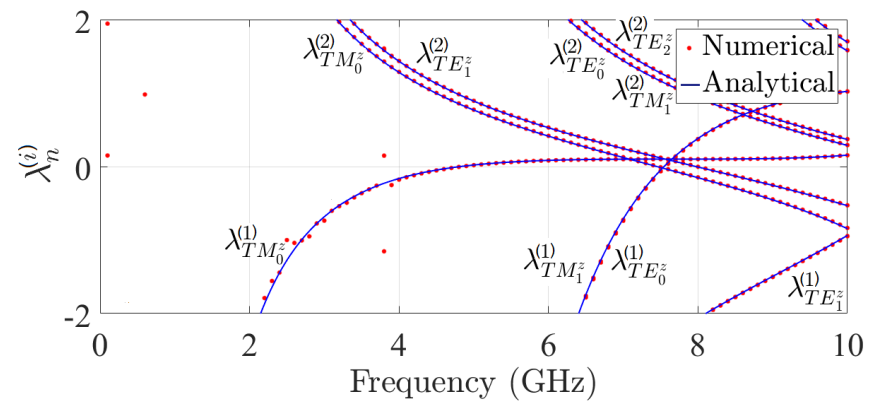

Fig. 2: Comparison between analytic and numerical characteristic eigenvalues for the infinite dielectric circular cylinder

$$
\left[\begin{array}{cc}
X & N_{i m} \\
N_{i m}^{t} & B
\end{array}\right]_{n}\left[\begin{array}{c}
J_{n}^{\lambda_{n}^{(i)}} \\
M_{n}^{\lambda_{n}^{(i)}}
\end{array}\right]=\lambda_{n}^{(i)}\left[\begin{array}{cc}
R & N_{r e} \\
N_{r e}^{t} & G
\end{array}\right]_{n}\left[\begin{array}{c}
J_{n}^{\lambda_{n}^{(i)}} \\
M_{n}^{\lambda_{n}^{(i)}}
\end{array}\right]
$$

In equation (11), $X, B, R, G, N_{i m}$ and $N_{r e}$ are the general tangential operators used from the PMCHWT formulation in [4]. It is important to emphasize that since each $n$-th mode has associated a system of two equations with two unknowns, it will involve two eigenvalues and two characteristic eigencurrents. These two solutions can be obtained for each $n$-th mode independently of the other modes solving (11), where $i=1,2$ is the index to distinguish the two solutions.

Now, let us consider as an example a non-magnetic cylinder of radius $a=5 \mathrm{~mm}$ and permittivity $\epsilon_{r_{2}}=9$. The outer media is assumed vacuum. See Fig. 1. Next, the characteristic eigenvalues of the cylinder will be studied to check whether the so-called nonphysical modes contribute to the electromagnetic field, and whether or not removing these solutions influence the accuracy of the resulting fields.

The characteristic eigenvalues of the cylinder are shown in Fig. 2. These analytical eigenvalues $\lambda_{n}^{(i)}$, corresponding to matrices (1) and (2), are obtained using (11). $\lambda_{n}^{(i)}$ are compared with those obtained numerically using FEKO [18]. Note that a 1-dimensional periodic boundary condition and the surface equivalence principle were used to simulate an infinite cylinder with FEKO. Fig. 2 shows an excellent agreement between the numerical and the analytical solutions. Those few points scattered outside the curve are due to purely numerical errors, since they do not fit with the curves obtained analytically.

As Fig. 2 shows, two types of curves can be clearly distinguished: curves with negative slope and curves with positive slope. Negative slope curves in this context are referred to as non-physical mode eigenvalues and positive slope curves as the physical ones. To distinguish one eigenvalue from the other, superscripts (1) and (2) in $\lambda_{n}^{(1)}$ and $\lambda_{n}^{(2)}$, have been used for physical and non-physical modes, respectively. Furthermore, each eigenvalue has its associated subscript indicating the polarization type and the order of the cylindrical harmonic solution, e.g., $T M_{0}^{z}$. It is interesting to see that eigenvalues 
corresponding to $T E_{0}^{z}$ and $T M_{1}^{z}$ coincide. Turning to the nonphysical modes, this term was adopted in [10] because they do not satisfy the far field orthonormality condition (12) associated to the scattered characteristic far fields $\mathbf{E}_{n}$ and $\mathbf{E}_{m}$.

$$
\frac{1}{\eta} \iint \mathbf{E}_{m} \mathbf{E}_{n}^{*} d s=\delta_{m, n}
$$

Rather than obtaining a value of unity when $m=n$, the researchers in [10] observed that the numerical evaluation of equation (12) on non-physical modes produced close-to-zero values instead.

However, this analytical study reveals that, at least for a 2D circular cylinder, an accurate computation of the far field under normal incidence leads to zero when computing equation (12) for all nonphysical modes and for any given frequency. These results have been obtained using equations (13) for $T M_{n}^{z}$ and (14) for $T E_{n}^{z}$ incidence.

$$
\begin{aligned}
E_{n}^{z}= & -\sqrt{\frac{j}{8 \pi k_{1}}} \frac{e^{-j k_{1} \rho}}{\sqrt{\rho}} \\
& \oint_{0}^{2 \pi}\left(\omega \mu_{0} \mathbf{J}_{n} \cdot \widehat{\mathbf{z}}-k_{1} \mathbf{M}_{n} \cdot \widehat{\boldsymbol{\phi}}\right) e^{-j k_{1} \rho^{\prime} \cos \left(\phi-\phi^{\prime}\right)} d \phi^{\prime} \\
E_{n}^{\phi}= & \sqrt{\frac{j}{8 \pi k_{1}}} \frac{e^{-j k_{1} \rho}}{\sqrt{\rho}} \\
& \oint_{0}^{2 \pi}\left(k_{1} \mathbf{M}_{n} \cdot \widehat{\mathbf{z}}+\omega \mu_{0} \mathbf{J}_{n} \cdot \widehat{\boldsymbol{\phi}}\right) e^{-j k_{1} \rho^{\prime} \cos \left(\phi-\phi^{\prime}\right)} d \phi^{\prime}
\end{aligned}
$$

In fact, what happens is that fields radiated by the equivalent electric and magnetic currents in (13) and (14) cancel each other for the nonphysical modes when $m=n$. So there is no field radiated by these non-physical modes. Therefore, as a first conclusion, it would be inappropriate to say that condition (12) is not met for the nonphysical modes since in reality there is no radiation at all from these modes.

As is well known [4], the scattered field can be expressed as the sum of the fields scattered by all characteristic currents. In view of previous result, the question arises about whether these modes contribute to the total field in the different regions involved and even whether the location of the source with respect to the dielectric body (inside or outside) may play a role in their contribution. As it will be demonstrated in the next section, the term non-physical may not be completely appropriate for these modes since it is found that they do contribute to the total electric field within the cylinder when the source is also within it.

\section{ON the Contribution to the Field of the Two PMCHWT EIGENSOLUTIONS.}

To understand better the eigencurrents associated to eigenvalues $\lambda_{n}^{(1)}$ and $\lambda_{n}^{(2)}$, it is necessary to go deeper into the formulation. The $i$ th eigenvector of equation (11) is related to the $i$-th eigenvalue. These eigenvectors form a weighted orthonormal set over the material body surface. Furthermore, the two eigenvectors of each $n$ mode combine to get the total induced current distribution when an excitation field is considered. Depending on the location of the source (internal or external to the surface of the cylinder), the $i=1$ and the $i=2$ eigenvectors will be excited or not. Each $i$-th eigenvector is a characteristic mode. An expression for the total induced current distribution as a function of the characteristic modes can be written as follows,

$$
\left[\begin{array}{c}
J \\
j M
\end{array}\right]=\sum_{n=-\infty}^{\infty} \sum_{i=1}^{2} \gamma_{i}^{n}\left[\begin{array}{c}
J_{n}^{\lambda_{n}^{(i)}} \\
j M_{n}^{\lambda_{n}^{(i)}}
\end{array}\right]\{\cos (n \phi), \sin (n \phi)\}
$$

where the excitation coefficients are

$$
\gamma_{i}^{n}=\frac{\left[\begin{array}{ll}
J_{n}^{\lambda_{n}^{(i)}} & j M_{n}^{\lambda_{n}^{(i)}}
\end{array}\right]\left[\begin{array}{c}
E^{i n c} \\
j H^{i n c}
\end{array}\right]}{\left(1+j \lambda_{n}^{(i)}\right)}
$$

Thus, the total current is given by the corresponding excited characteristic modes given by the two $i$-th solutions. Therefore both $i$-th eigenvectors and eigenvalues have to be taken into account. The analytical results for the total current distribution considering equation (15) for an external or an internal incident field are presented below.

On the one hand, Figs. $3 \mathrm{a}$ and $3 \mathrm{~b}$ show the total electric and magnetic equivalent currents obtained for a $T M^{z}$ plane wave impinging normally on the dielectric cylinder surface at $f=4 \mathrm{GHz}$. To calculate the total current distribution using equation (15), the incident plane wave was written in terms of cylindrical wave functions.

$$
\begin{aligned}
\mathbf{E}^{i n c} & =E_{0} \sum_{n=-\infty}^{+\infty} j^{-n} J_{n}\left(k_{1} \rho\right) e^{j n \phi} \widehat{z} \\
\mathbf{H}^{i n c} & =E_{0} / \eta_{1} \sum_{n=-\infty}^{+\infty} j^{-(n+1)} J_{n}^{\prime}\left(k_{1} \rho\right) e^{j n \phi} \widehat{\phi}
\end{aligned}
$$

Either in Fig. $3 a$ or $3 b$, the contribution of the non-physical solution to the total current is found to be exactly zero. It can be seen that when the $T M^{z}$ excitation is considered, only the solution with $i=1$ contributes to the total current distribution. Although obvious, it is important to point out that since the contribution of non-physical modes to the total current is zero, their contribution to the electromagnetic field will equally be zero, either inside or outside the cylinder.

Let us consider now an electric line source within the cylinder. The source is located along the z-axis for simplicity. As in the example above, equation (15) is used, but now the incident electric and magnetic fields are,

$$
\begin{aligned}
\mathbf{E}^{i n c} & =-\frac{k_{2} \eta_{2} I}{4} H_{0}^{(2)}\left(k_{2} \rho\right) \widehat{z} \\
\mathbf{H}^{i n c} & =\frac{k_{2} I}{4 j} H_{1}^{(2)}\left(k_{2} \rho\right) \widehat{\phi}
\end{aligned}
$$

The goal is to know whether non-physical modes are excited or not. Since the excitation is rotationally symmetric, so are the equivalent currents and only $T M_{0}^{z}$ mode contributes to the total field. Table I shows amplitude and phase of the total electric and magnetic equivalent currents obtained for this case. Results were obtained for a frequency of $4 \mathrm{GHz}$. Unlike for a plane wave, in this case both characteristic mode solutions, $i=1$ and $i=2$, do contribute to total equivalent currents. Both modes are meaningful under this sort of excitation. Letter $X$ in Table I is used to simplify notation and stands for electric, $J$, or magnetic current, $M$.

Now, the electric field radiated by the physical $(i=1)$ and nonphysical $(i=2)$ solutions produced by the currents in Table I are obtained. Equations (21) and (22) are the scattered outer and inner electric fields, respectively. These fields are obtained in terms of the characteristic modes $i=1$ and $i=2$. 


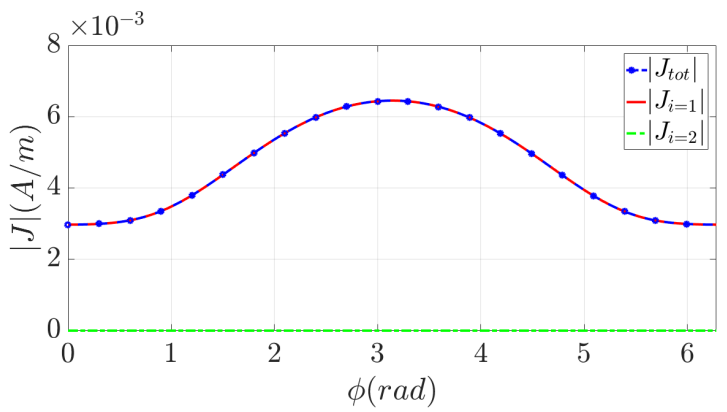

(a)

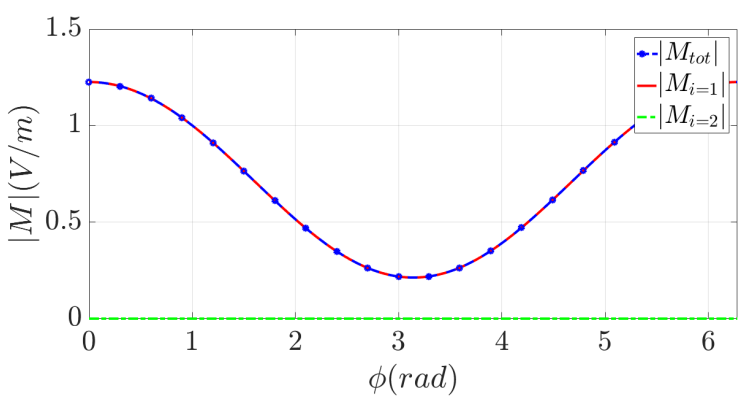

(b)

Fig. 3: Contribution of each eigensolution when impinging by a normally incident TMz plane wave: (a) To the total electric current. (b) To the total magnetic current.

TABLE I: Contribution of each eigensolution to the total electric and magnetic current for an electric line source excitation in $\mathrm{z}$-axis.

\begin{tabular}{|c|c|c|}
\hline & $\begin{array}{c}\text { Electric current, } X=\mathbf{J} \\
{[\text { Abs }(\mathbf{A} / \mathbf{m}), \text { Phase (rad) }]}\end{array}$ & $\begin{array}{c}\text { Magnetic current, } \\
=\mathbf{M}[\text { Abs }(\mathbf{V} / \mathbf{m}), \\
\text { Phase (rad) }]\end{array}$ \\
\hline \hline$\gamma_{1}^{0} X_{0}^{\lambda_{0}^{(1)}}$ & {$[\mathbf{2 . 8 7 3 2 e + 1 0 , 2 . 9 1 4 8 ]}$} & {$[\mathbf{4 . 5 2 1 1 e + 1 2 , 1 . 3 4 4 1 ]}$} \\
\hline$\gamma_{2}^{0} X_{0}^{\lambda_{0}^{(2)}}$ & {$[\mathbf{3 . 1 8 6 1 e + 0 8}, \mathbf{1 . 8 4 5 4}]$} & {$[\mathbf{5 . 6 0 0 4 e + 1 1 , ~ 0 . 2 7 4 6 ]}$} \\
\hline$\sum_{i=1}^{2} \gamma_{i}^{0} X_{0}^{\lambda_{0}^{(i)}}$ & {$[\mathbf{2 . 8 8 8 7 e + 1 0}, \mathbf{2 . 9 0 5 2}]$} & {$[\mathbf{4 . 8 1 5 4 e + 1 2 , 1 . 2 4 1 9 ]}$} \\
\hline
\end{tabular}

$$
\begin{aligned}
E_{\text {scat }, \text { out }}^{z}= & \frac{\eta_{1} \pi k_{1} a}{2} J_{0}\left(k_{1} a\right) H_{0}^{(2)}\left(k_{1} \rho\right) \sum_{i=1}^{2} \gamma_{i}^{0} J_{0}^{\lambda_{0}^{(i)}} \\
& +\frac{k_{1} \pi a}{2 j} J_{1}\left(k_{1} a\right) H_{0}^{(2)}\left(k_{1} \rho\right) \sum_{i=1}^{2} \gamma_{i}^{0} M_{0}^{\lambda_{0}^{(i)}} \\
E_{\text {scat }, \text { in }}^{z}= & -\frac{\eta_{2} \pi k_{2} a}{2} J_{0}\left(k_{2} \rho\right) H_{0}^{(2)}\left(k_{2} a\right) \sum_{i=1}^{2} \gamma_{i}^{0} J_{0}^{\lambda_{0}^{(i)}} \\
& -\frac{k_{2} \pi a}{2 j} J_{0}\left(k_{2} \rho\right) H_{1}^{(2)}\left(k_{2} a\right) \sum_{i=1}^{2} \gamma_{i}^{0} M_{0}^{\lambda_{0}^{(i)}}
\end{aligned}
$$

Figs. $4 \mathrm{a}, 4 \mathrm{~b}$ and $4 \mathrm{c}$ show these fields graphically as a function of the radial distance to the origin, $\rho$. Fig. 4a shows the total electric field. It includes the contribution of the line source and the equivalent currents. As expected, the field is continuous at the surface interface, $\rho=5 \mathrm{~mm}$. Figs. $4 \mathrm{~b}$ and $4 \mathrm{c}$ show the scattered electric field generated by the equivalent currents $i=1$ and $i=2$, respectively. Notice

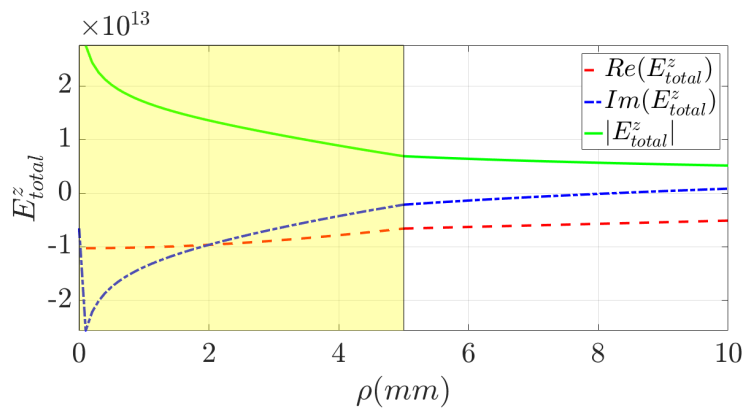

(a)

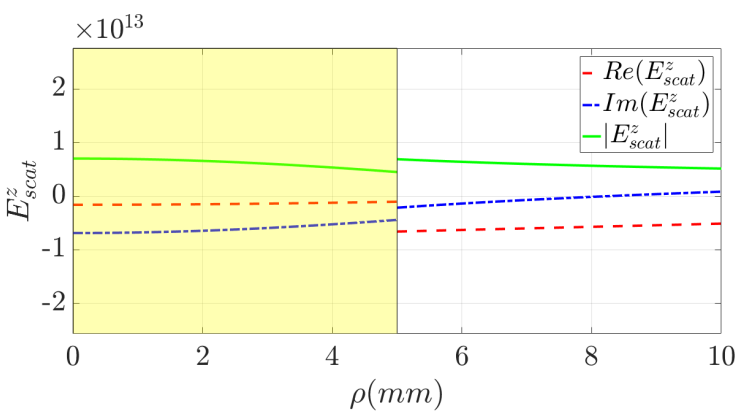

(b)

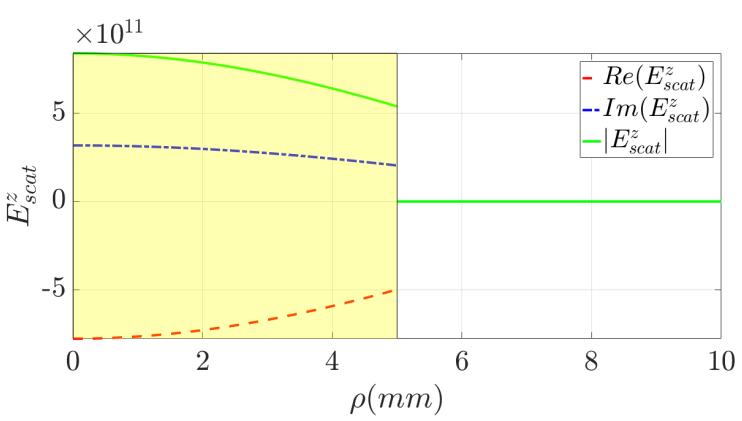

(c)

Fig. 4: Electric field contribution inside and outside the cylinder by the two equivalent currents when fed by an electric line source in the z-axis ( $f=4 \mathrm{GHz}, a=5 \mathrm{~mm}, \epsilon_{r_{2}}=9$ ): (a) Total electric field . (b) Scattered field due to the $i=1$ equivalent currents. (c) Scattered field due to the $i=2$ equivalent currents (non-physical modes solution).

that a different scale was used in Fig. 4c for clarity. It is worth noticing that the outer scattered field radiated by the equivalent current corresponding to $i=2$ is zero. This current only contributes to the inner scattered field, $\rho<5 \mathrm{~mm}$.

For the sake of completeness, it is also interesting to show the behavior of the magnetic field radiated by the physical $(i=1)$ and nonphysical $(i=2)$ solutions produced by the currents in Table I, as done for the electric field. Equations (23) and (24) are the scattered outer and inner magnetic fields, respectively. These fields are obtained in terms of the characteristic modes $i=1$ and $i=2$. 


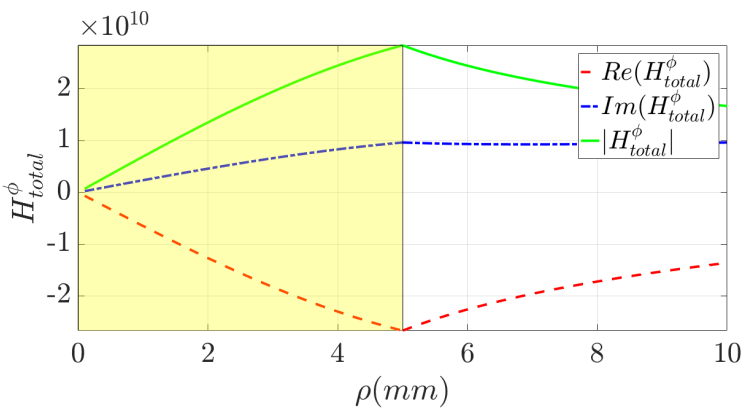

(a)

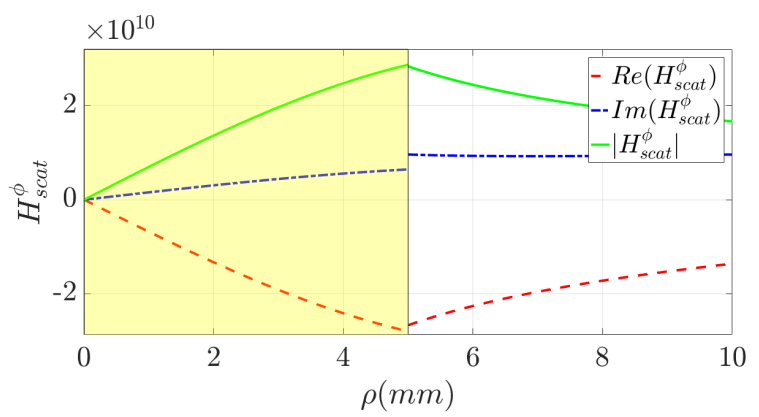

(b)

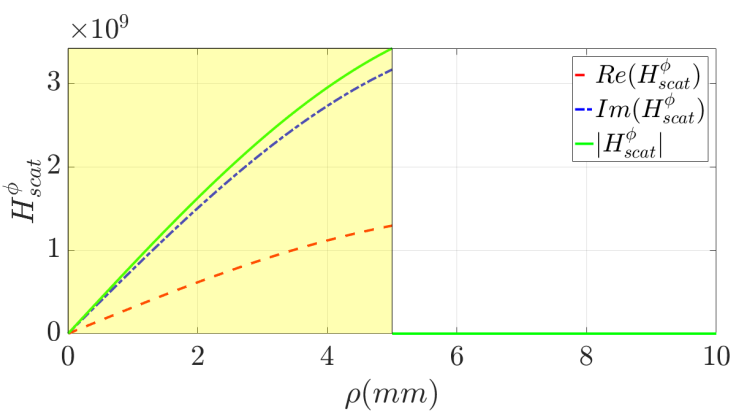

(c)

Fig. 5: Magnetic field contribution inside and outside the cylinder by the two equivalent currents when fed by an electric line source in the z-axis ( $f=4 \mathrm{GHz}, a=5 \mathrm{~mm}, \epsilon_{r_{2}}=9$ ). (a) Total magnetic field. (b) Scattered field due to the $i=1$ equivalent currents. (c) Scattered field due to the $i=2$ equivalent currents (non-physical modes solution).

$$
\begin{aligned}
H_{\text {scat }, \text { out }}^{z} & =-\frac{\pi k_{1} a}{2 j} J_{0}\left(k_{1} a\right) H_{0}^{\prime(2)}\left(k_{1} \rho\right) \sum_{i=1}^{2} \gamma_{i}^{0} J_{0}^{\lambda_{0}^{(i)}} \\
& +\frac{k_{1} \pi a}{2 \eta_{1}} J_{1}\left(k_{1} a\right) H_{0}^{\prime(2)}\left(k_{1} \rho\right) \sum_{i=1}^{2} \gamma_{i}^{0} M_{0}^{\lambda_{0}^{(i)}} \\
H_{\text {scat }, \text { in }}^{z} & =\frac{\pi k_{2} a}{2 j} J_{0}^{\prime}\left(k_{2} \rho\right) H_{0}^{(2)}\left(k_{2} a\right) \sum_{i=1}^{2} \gamma_{i}^{0} J_{0}^{\lambda_{0}^{(i)}} \\
& -\frac{k_{2} \pi a}{2 \eta_{2}} J_{0}^{\prime}\left(k_{2} \rho\right) H_{1}^{(2)}\left(k_{2} a\right) \sum_{i=1}^{2} \gamma_{i}^{0} M_{0}^{\lambda_{0}^{(i)}}
\end{aligned}
$$

The same behaviour can be observed for the magnetic field. Figs. $5 \mathrm{a}, 5 \mathrm{~b}$ and $5 \mathrm{c}$ show these fields graphically as a function of the radial distance to the origin, $\rho$.

Following the discussion in previous section, these results support the motive for which the so-called non-physical modes do not comply with the orthonormality condition (12). There is no electromagnetic radiation from non-physical characteristic mode outside the cylinder surface. Therefore they should be named more properly as nonradiating modes instead, since they do contribute to inner fields when source is inside.

These analytical results allow us to understand better the characteristic eigenvalues obtained numerically from the PMCHWT formulation in previous studies, when analyzing an infinite dielectric circular cylinder. These conclusions are independent of frequency, material or radius used for the cylinder.

\section{CONCLUSION}

The object of this communication has been to analyze accurately and to clarify the underlying physics of the characteristic mode solutions provided by the PMCHWT formulation for dielectric bodies. For that purpose, an infinite dielectric circular cylinder has been selected. For such canonical problem, an analytical solution can be provided, cutting out from the results interpretation any shadow associated to numerical errors. Thanks to that, it has been possible to demonstrate that all solutions provided by the PMCHWT formulation have indeed a physical meaning. This was necessary since, to date, there has been doubts on certain mathematical solutions of this formulation which were classified as non-physical. In this regard it is worth mentioning that the electromagnetic suite FEKO [18] currently eliminates these modes from the solution presented to the user. In reality so-called non-physical modes do contribute to the field within the cylinder when the excitation is also in it. Therefore it would be more appropriate to qualify them as non-radiating modes.

It is important to emphasize that the results obtained in this contribution do not contradict the key finding in [10] regarding the non-radiating nature of their non-physical modes for 3D arbitrary geometries. In fact it supports it and clarifies the cause behind these modes not satisfying the field orthogonality relation. Likewise, it is reasonable to infer that non-radiating modes will contribute too to the field within a 3D arbitrary structure when the source is within it, as they do for the cylinder, since the cause of it lays on the properties of the PMCHWT integro-differential operator, not in the geometry itself. Certainly this statement will require a numerical proof but this is left for future work.

\section{REFERENCES}

[1] R. J. Garbacz, "A generalized expansion for radiated and scatered fields," Ph.D. dissertation, The Ohio State University, Columbus, 1968.

[2] R. Harrington and J. Mautz, "Theory of characteristic modes for conducting bodies," Antennas and Propagation, IEEE Transactions on, vol. 19, no. 5, pp. 622-628, 1971.

[3] R. Harrington, J. Mautz, and Y. Chang, "Characteristic modes for dielectric and magnetic bodies," Antennas and Propagation, IEEE Transactions on, vol. 20, no. 2, pp. 194-198, 1972.

[4] Y. Chang and R. Harrington, "A surface formulation for characteristic modes of material bodies," Antennas and Propagation, IEEE Transactions on, vol. 25, no. 6, pp. 789-795, 1977.

[5] M. Cabedo Fabres, "Systematic design of antennas using the theory of characteristic modes," Ph.D. dissertation, U. Politècnica de València, Valencia, 2008.

[6] E. A. Daviu, "Analysis and design of antennas for wireless communications using modal methods," Ph.D. dissertation, Universitat Politècnica de València, Valencia, 2008.

[7] M. Cabedo-Fabres, E. Antonino-Daviu, A. Valero-Nogueira, and M. F. Bataller, "The theory of characteristic modes revisited: A contribution to the design of antennas for modern applications," Antennas and Propagation Magazine, IEEE, vol. 49, no. 5, pp. 52-68, 2007.

[8] F. Gallée, T. Bernabeu-Jiménez, M. Cabedo-Fabres, E. Antonino-Daviu, A. Valero-Nogueira, and C. Person, "Conception d'une antenne de type dra à 60ghz avec la théorie des modes caractéristiques," JNM 2013: 18èmes Journées Nationales Microondes, 2013. 
[9] T. Bernabeu-Jimenez, A. Valero-Nogueira, F. Vico-Bondia, E. Antonino-Daviu, and M. Cabedo-Fabres, "A 60-ghz ltcc rectangular dielectric resonator antenna design with characteristic modes theory," in Antennas and Propagation Society International Symposium (APSURSI), 2014 IEEE. IEEE, 2014, pp. 1928-1929.

[10] H. Alroughani, J. L. Ethier, D. McNamara et al., "Observations on computational outcomes for the characteristic modes of dielectric objects," in Antennas and Propagation Society International Symposium (APSURSI), 2014 IEEE. IEEE, 2014, pp. 844-845.

[11] _ "On the classification of characteristic modes, and the extension of sub-structure modes to include penetrable material," in Electromagnetics in Advanced Applications (ICEAA), 2014 International Conference on. IEEE, 2014, pp. 159-162.

[12] Y. Chen and C.-F. Wang, "Surface integral equation based characteristic mode formulation for dielectric resonators," in Antennas and Propagation Society International Symposium (APSURSI), 2014 IEEE. IEEE, 2014, pp. 846-847.

[13] R. Maximidis, C. Zekios, T. N. Kaifas, E. Vafiadis, and G. Kyriacou, "Characteristic mode analysis of composite metal-dielectric structure, based on surface integral equation/moment method," in Antennas and Propagation (EuCAP), 2014 8th European Conference on. IEEE, 2014 pp. 2822-2826.

[14] T. Bernabeu Jimenez, A. Valero Nogueira, F. Vico Bondia, A. Vila Jimenez, D. Sanchez Escuderos, and F. Gallee, "A 60-ghz coplanar-waveguide-fed slot-coupled rectangular dra design using the theory of characteristic modes," in Antennas and Propagation (EuCAP), 2015 9th European Conference on. IEEE, 2015.

[15] A. J. Poggio and E. K. Miller, Integral equation Solution of Threedimensional Scattering Problems, Computer techniques in electromagnetics, R. Mittra. Pergamon Press, Oxford, pp. 159-261, 1973.

[16] H. Alroughani, "An appraisal of the characteristic modes of composite objects," Master thesis, University of Ottawa, 2013.

[17] Y. Chang and R. F. Harrington, "A surface formulation for characteristic modes of material bodies," DTIC Document, Tech. Rep., 1974.

[18] FEKO Suite 7.0, Altair Development S.A. (Pty), Stellenbosch, South Africa, http://www.altairhyperworks.com. 